\title{
A Computational Analysis of the Interfacial Curvature Effect on the Strength of a Material with a Modified Surface Layer
}

\author{
Ruslan R. Balokhonov ${ }^{1,2, a)}$, Sergei A. Martynov ${ }^{1, b)}$, Varvara A. Romanova ${ }^{1,2, c)}$, \\ and Aleksandr V. Zinoviev ${ }^{1, d)}$ \\ ${ }^{1}$ Institute of Strength Physics and Materials Science SB RAS, Tomsk, 634055, Russia \\ ${ }^{2}$ National Research Tomsk Polytechnic University, Tomsk, 634050, Russia \\ a) Corresponding author: rusy@ispms.ru \\ b) martynov@ispms.tsc.r \\ c) varvara@ispms.ru \\ d) zav@ispms.ru
}

\begin{abstract}
The mechanisms of the deformation and fracture of coated materials with varying coating thickness and coating-substrate interfacial curvature parameters are investigated. The boundary-value problem in the plane strain formulation is solved numerically, using the finite-difference method. In the calculations, an explicit account is taken of experimental and model microstructures with irregular and sinusoidal coating-substrate interface geometries, respectively. The stress concentration in the near-interface region is shown to increase with decrease in the coating thickness and increase in the sinusoidal interface amplitude. This dependence is nonlinear in character.
\end{abstract}

Keywords: mechanics of inhomogeneous media, numerical simulation, composites, coated and surface-hardened materials

\section{INTRODUCTION}

Different surface treatment and coating deposition technologies allow for modified hardened layers and highstrength coatings with a curvilinear profile of the coating-substrate interface to be produced on the material surface (Fig. 1).

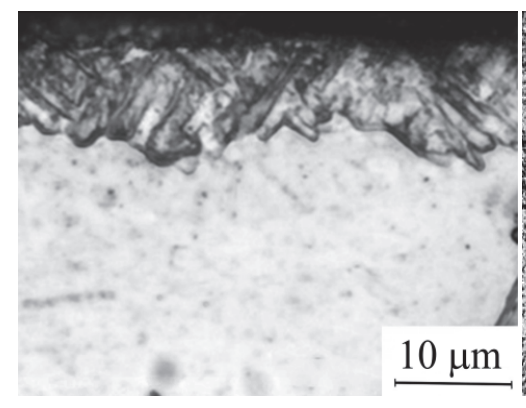

(a)

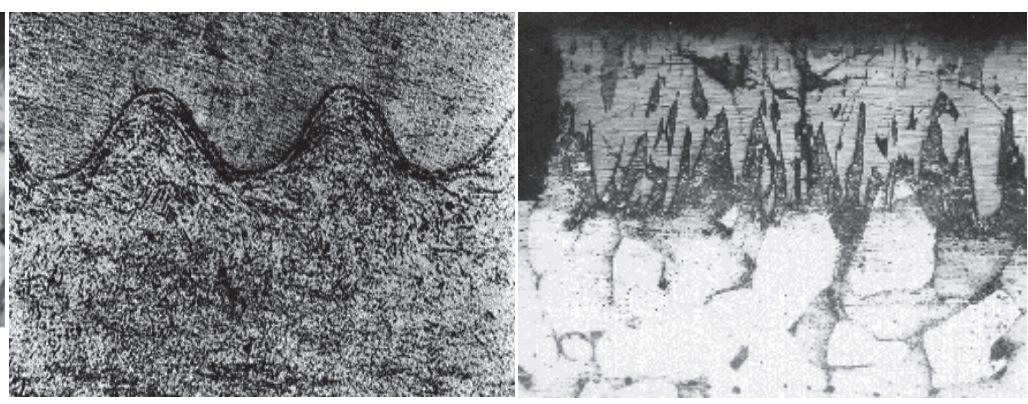

(b)

(c)

FIGURE 1. Experimental microstructures containing wavy interfaces produced by means of a LEHCEB surface treatment of a commercial titanium VT1-0 substrate (a) [1], explosion Ti-Ti welding (b) [2] and diffusion borating of a steel substrate (c) [3] 


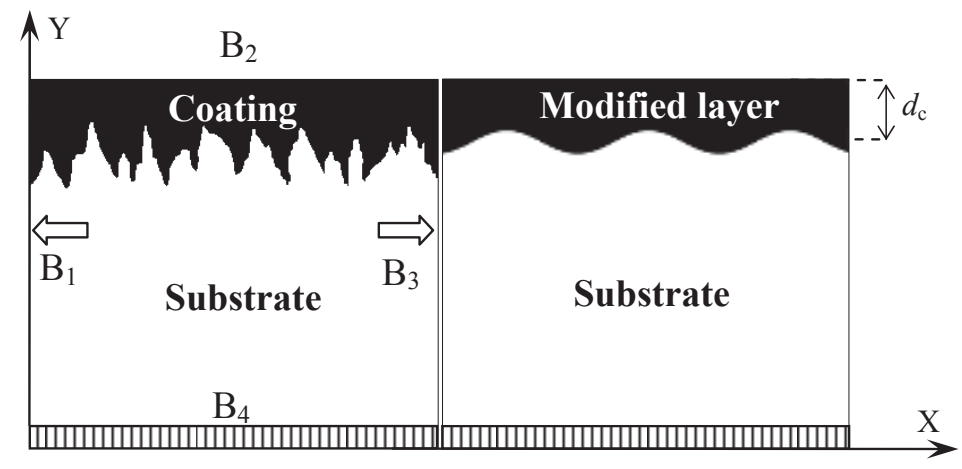

(a)

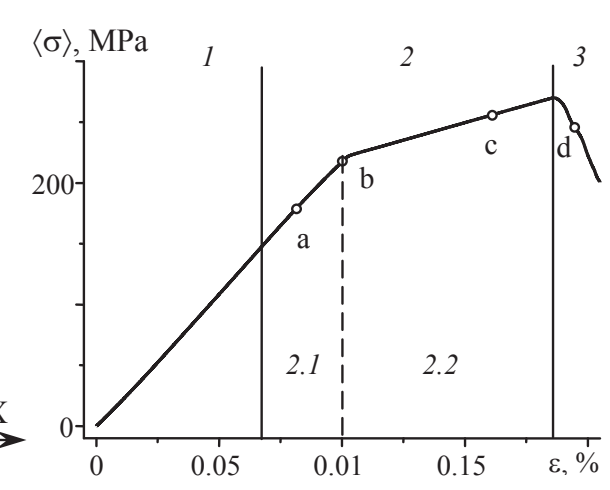

(c)

FIGURE 2. Model microstructures with toothed (a) and sinusoidal coating-substrate interfaces (b) and the stress-strain curve under tension (c)

For instance, a low-energy high-current electron beam (LEHCEB) treatment leads to melting followed by fast crystallization of a thin surface layer of commercial titanium VT1-0 samples [1]. The microstructure and thickness of the modified surface layer depend on the energy density, with the interfacial geometry being of a wavy sinusoidal shape (Fig. 1(a)). Explosion welding [2] and diffusion borating [3] produce sinusoidal wavy (Fig. 1(b)) and toothed wavy interfacial profiles (Fig. 1(c)), respectively.

\section{STATEMENT OF THE PROBLEM AND SIMULATION RESULTS}

The boundary-value dynamic problem on the substrate-coating tension was solved numerically [4]. Constitutive models of the elastic-plastic behavior and elastic-brittle fracture [4,5] were used to describe the mechanical responses of the substrate and coating materials. The wavy interfacial curvature of the needle-like and sinusoidal types was simulated. The pertinent simplified microstructures are shown in Figs. 2(a, b), respectively. The macroscopic stress-strain curve for the microstructure presented in Fig. 2(a) is shown in Fig. 2(c). Three main deformation stages are clearly seen in the curve: 1 - elasticity, 2-plasticity and 3-fracture. At stage 1, both the substrate and the coating are deformed elastically. Due to the difference in the elastic moduli, the stress-strain state is inhomogeneous, with local stress concentrations being distributed over the coating along the coating-substrate interface. Stage 2 involves plastic deformation in the substrate, whereas the coating is in the elastic state. The plastic deformation stage, in its turn, can be divided into two substages. At substage 2.1, plastic strains are localized in the near-interface regions, while a major part of the substrate is still in an elastic state (Figs. 3(a, b)). The portion of the stress-strain curve corresponding to substage 2.1 demonstrates a near-linear behavior similar to that observed at the elastic stage. At substage 2.2, a major part of the substrate transforms into a plastic state (Fig. 3(c)), and the slope of the stress-strain curve changes (Fig. 1(c)).

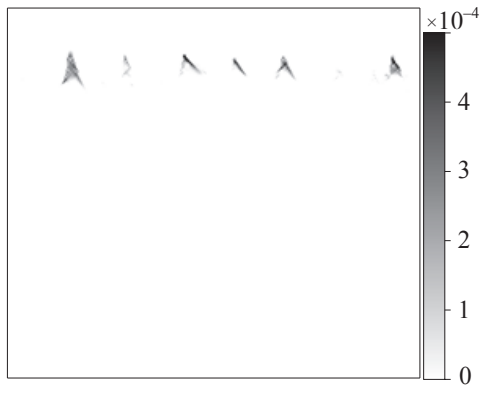

(a)

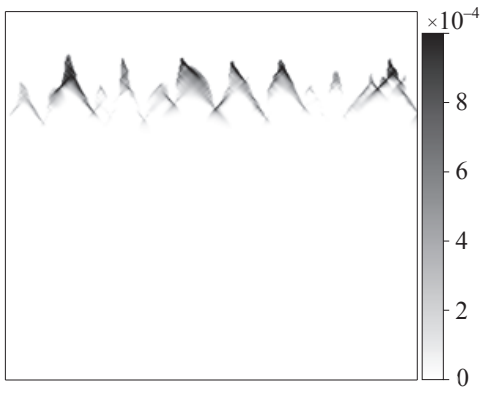

(b)

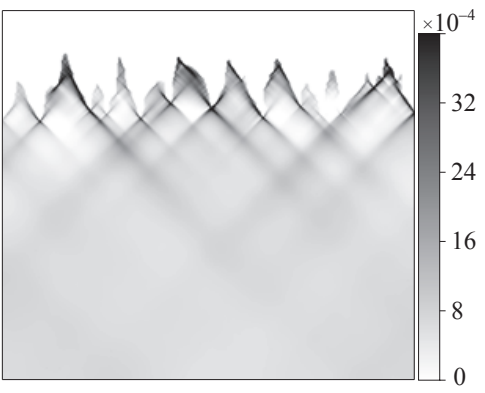

(c)

FIGURE 3. Equivalent plastic strain patterns for the states a-c presented in Fig. 2 (b): $\varepsilon=0.08 \%$ (a), $0.1 \%$ (b) and $0.16 \%$ (c) 


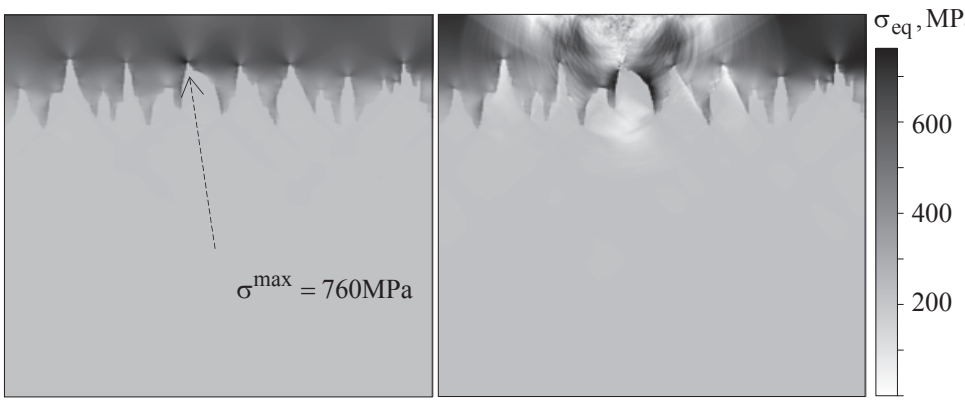

(a)

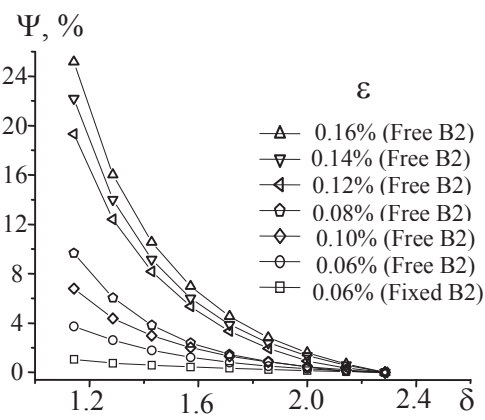

(c)

FIGURE 4. Equivalent stress patterns (a) and (b) for the states $\mathrm{c}$ and d presented in Fig. 1(c) and the maximum relative equivalent stress concentration vs. the relative coating thickness at different elongation stages in the coated material (c)

A maximum stress concentration region near the coating-substrate interface is shown in Fig. 4(a). It is in this location where fracture will occur at stage 3 (Fig. 4(b)).

In the calculations, the average coating thickness $d_{\mathrm{c}}$ (Fig. 1) was varied, with the interfacial geometry being the same. The maximum stress concentration in the near-interface region was found to decrease nonlinearly with increase in the coating thickness and to saturate. On further increase in $d_{\mathrm{c}}$ the stress changes but only slightly (Fig. 4(c)). Let us investigate the coating thickness effect at different deformation stages in the coated material and consider the relative stress concentration to obtain $\Psi=\frac{\sigma^{\max }-\sigma_{\text {sat }}^{\max }}{\sigma_{\text {sat }}^{\max }} \cdot 100 \%$, where $\sigma_{\text {sat }}^{\max }$ is the maximum saturated equivalent stresses at varying coating thickness. Let us consider the relative thickness $\delta=d_{\mathrm{c}} / d_{\mathrm{t}}$, where $d_{\mathrm{c}}$ is the coating thickness and $d_{\mathrm{t}}$ is the average thickness of the toothed coating sublayer (Fig. 2(a)).

The coating thickness effect is observed both at the elastic deformation stage 1 and at the plastic flow stage 2 (Fig. 4(c)). As compared with the case where the boundary $B_{2}$ (Fig. 1(a)) is a free surface, the maximum stress is found to decrease slower by a factor of 3 for the case where the boundary is fixed in direction $Y$ (cf. the free $B_{2}$ and fixed $B_{2}$ cases for $\varepsilon=0.06 \%$ in Fig. $4(\mathrm{c})$ ).

The maximum coating thickness effect accounts for $4 \%$ and $25 \%$ at the elastic and pre-fracture deformation stages in the coated material, respectively (Fig. 4(c)).

The dependence shown in Fig. 4(c) is invariable to the physical size of the microstructure (Fig. 1(a)) subjected to quasi-static loading. This means that for given microstructural geometry and mechanical properties of the substrate and coating materials, optimum thickness of the coating is 2-2.5 times as great as the roughness of the coatingsubstrate interface. The roughness of the interface is likely to depend on that of the substrate to be coated. This assumption can be used in experimental investigations and industrial applications to determine optimum coating thickness.

As is shown above, the maximum stress concentration depends on two key parameters: the coating thickness and the interfacial curvature. In addition to the coating thickness effect, the influence of the interfacial curvature was studied in detail for the microstructure of the coated material with a perfect sinusoidal coating-substrate interface presented in Fig. 2(b). Figure 5(a) shows a representative fragment of the microstructure. A series of numerical simulations was performed for a microstructure under tension, with the sinusoidal amplitude $A$ being varied from 10 to $120 \mu \mathrm{m}$. To avoid the coating thickness effect, the same thickness of the continuous coating layer was used in the calculations $(\delta \approx 6)$ which is several times greater than the optimum value $\delta \approx 2.4$ (Fig. 4(c)).

The dependence of the maximum relative equivalent stress concentration on the sinusoidal amplitude is shown in Fig. 5(b) at different stages of the microstructural elongation. Two main conclusions can be drawn on the basis of the calculation results. First, the higher is the elongation, the more similar to each other are the shapes of the curves, i.e., the amplitude effect on the strength of the coated material is the same late in the deformation of the material (see the curves in Fig. 5(b) for $\varepsilon>0.7 \%$ ). This is due to the plastic strain localization at substage 2.1 discussed above and illustrated in Figs. 3( $a$ and $b)$. 


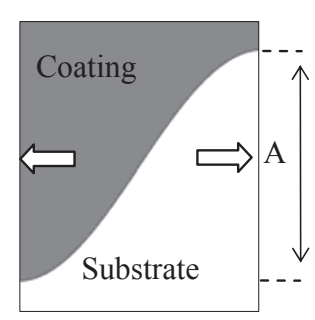

(a)

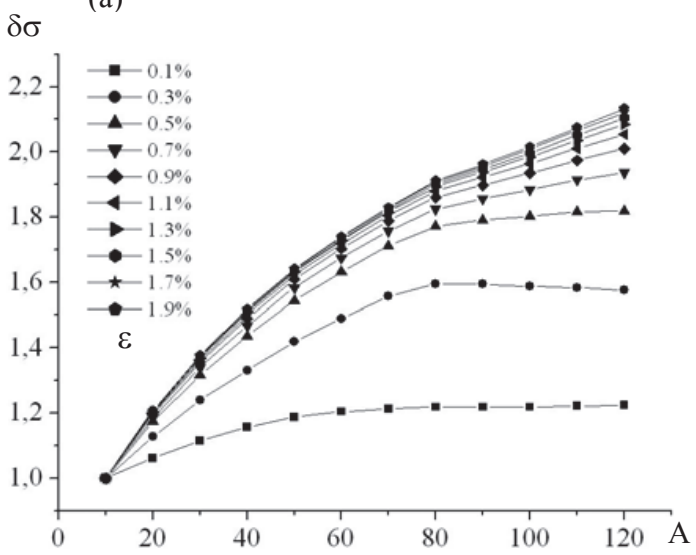

(b)

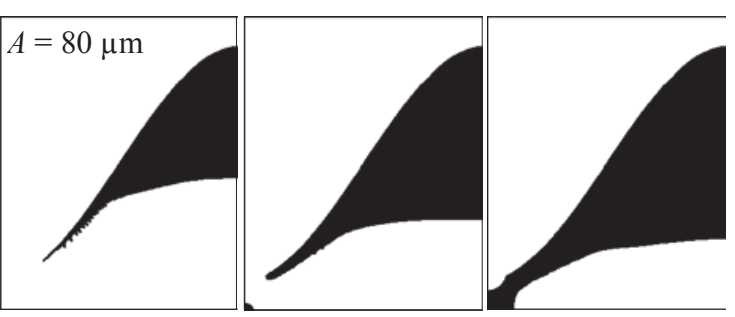

(c)

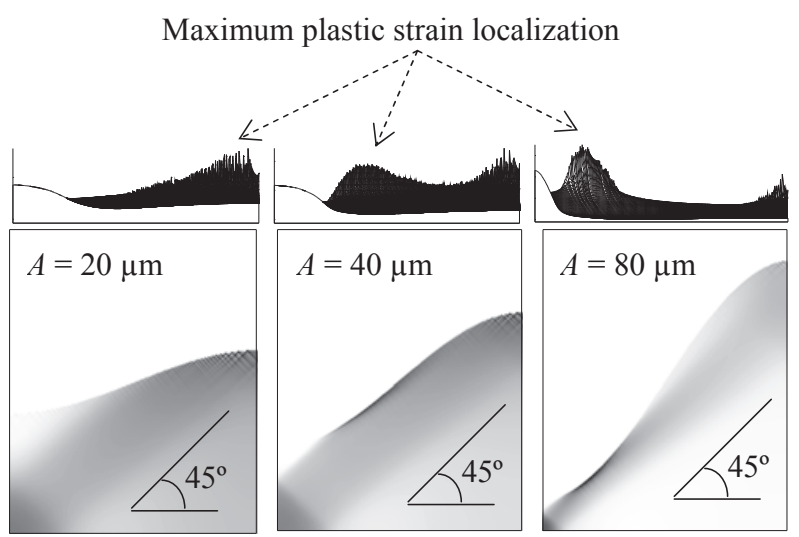

(d)

FIGURE 5. Local microstructure with a sinusoidal coating-substrate interface (a), maximum relative stress concentration vs. sine curve amplitude $A(\mathrm{~b})$, black regions represent plastic flow for different elongation strains acting on the microstructure with $A=80 \mu \mathrm{m}$ (c) and plastic strain patterns for $A=20,40$ and $80 \mu \mathrm{m}$ and the elongation strain $\varepsilon=0.3 \%$ (d)

The curves in Fig. 5(b) for $\varepsilon<0.7 \%$ differ from each other during the strain localization process in the case where the plastic strains originate near the sinusoidal interface and propagate deep into the bulk, covering the whole of the substrate material (Fig. 5(c)). Second, the slope of the curves changes for a sinusoidal amplitude of about 80 $\mu \mathrm{m}$. This is due to different locations of the maximum plastic strain in the near-interface region for different sinusoidal amplitudes (Fig. 5(d)), which is connected with the angle between the interfacial profile and the direction of the maximum tangential stress. At low and high amplitudes $(A=20$ and $80 \mu \mathrm{m}$ in Fig. 5(d)), the plastic strain is localized at the top and at the bottom of the interface, respectively, while the center of the interface is the strain localization point for a moderate amplitude in the case where the interface is at about $45^{\circ}$ to the direction of load application, i.e. it is aligned with the direction of the maximum tangential stress $(A=40 \mu \mathrm{m}$ in Fig. 5(d)).

The support of Russian Science Foundation (grant No. 14-19-00766) and a program to increase the competence of TPU is gratefully acknowledged.

\section{REFERENCES}

1. A. V. Panin, M. S. Kazachenok, O. M. Kretova, O. B. Perevalova, Y. F. Ivanov, A. M. Lider, O. M. Stepanova, and M. H. Kroening, Appl. Surf. Sci. 284, 750 (2013).

2. Y.A. Conon, L. B. Pervukhin, and A.D. Chudnovsky, Explosion Welding, Mechanical Engineering (Mosvow, 1987).

3. A. V. Koval and S. V. Panin, Theor. Appl. Fract. Mech. 34, 117 (2000).

4. R. R. Balokhonov, V. A. Romanova, S. Schmauder, S. A. Martynov, and Zh. G. Kovalevskaya, Compos. Part B Eng. 66, 276 (2014).

5. R. R. Balokhonov, V. A. Romanova, and S. Schmauder, Comp. Mater. Sci. 37, 110 (2006). 